\title{
Commentary
}

\section{Estimating fitness from offspring counts in clonal seed plants}

\author{
Lonnie W. Aarssen \\ Lonnie W. Aarssen (aarssenl@queensu.ca), Department of Biology, Queen's University, Kingston, ON, Canada, \\ K7L $3 N 6$
}

\begin{abstract}
Should asexual/vegetative offspring be counted in estimates of evolutionary fitness in seed plants? The answer it seems remains debatable among evolutionary ecologists. Arguably, however, the answer is yes when fitness is ascribed strictly to resident genes/alleles, in terms of their survival-through copying and transmission success-over time. This success results only because sporophyte 'rooted units' make more 'rooted units' - not primarily in terms of leaving descendent 'individuals', but more fundamentally as carriers of gene/allele copies in perpetuity. The 'rootedunit' (RU) is identified by root and shoot tissue integrated through an essential, and distinctly local, vascular transition. It thus represents a functional and practical circumscription for enumerating individual 'offspring' that applies equally to those derived from zygotes, or from clonality. This 'binary' nature of fitness estimation in seed plants remains legitimate despite that clonal RU offspring are normally all genotypically identical; the same is true of zygotic/seed-derived RU offspring produced by apomixis or from inbred sexual lines (and somatic mutation sometimes means that clonal offspring are not, in fact, genotypically identical). Neither is this fitness estimation compromised if a young clonal RU offspring, during establishment, temporarily receives resource translocation from connection to a parental RU; the same is true for immature zygotic offspring that receive maternal resources within the seed, via female gametophyte or endosperm/ perisperm tissue. Finally, the above fitness estimate also remains uncompromised even if there is facilitation between mature neighbouring RUs that remain
\end{abstract}

connected with lifetime physiological integration; analogous facilitation also occurs routinely between neighbouring RUs in aclonal species.

Keywords: clonal plants, fitness, genet, individual, offspring, ramet, rooted-unit

The relationship between clonality and the definition of an 'individual' or an 'offspring' in plants was a contentious subject for evolutionary biology in earlier decades (e.g. reviewed by Mogie 1992). But the issue it seems was never really resolved, and recent discussions have sparked renewed debate (Clarke 2012, Gorelick 2012). A central question for seed plants is whether evolutionary fitness should be estimated only from counts of sporophyte offspring derived from zygotes (seeds), or whether clonal (asexual/vegetative) offspring can/should also be counted (Fagerstrom 1992, Pan and Price 2002). The uncertainty extends also to decisions regarding what units represent demographic 'individuals' for counting in population sampling for abundance or recruitment success. The aim of the present commentary, therefore, is to briefly revisit a supporting argument for including clonal offspring/individuals, with anticipation of generating extended conversation for exploring whether it is possible to arrive at a broader and more conspicuous consensus - either way-among researchers. Progress for this, in my view, may depend on greater (and more conspicuous) consensus regarding whether fitness is meaningfully ascribed to individual plants, or only to the genes/alleles that they carry (sensu Dawkins 1989). 


\section{Offspring as 'rooted-units'}

Zygotic (seed-derived) offspring develop through mitotic cell divisions involving iterative production and differentiation of totipotent meristems, with the collective product sometimes referred to as a 'genet' (Harper 1977). In terrestrial spermatophtyes, this product virtually always involves at least one 'rooted unit' (RU), and only one (by definition) in aclonal species (Figure 1a). A RU is identified by a local root-shoot vascular transition (normally involving distinctly different aboveand below-ground stele configurations of xylem and phloem tissue), and where the associated root or shoot tissue - or both together-is/are laterally discontinuous with other such transitions (Figure 1). In aclonal species, both the shoot and root tissue products of a single zygote (genet) are always permanently, and entirely, laterally discontinuous between rooted-units (because 1 genet = 1 RU) (Figure 1a). Clonal species, however, have capacity for an important variation: a rooted unit can have only shoot tissue that is laterally discontinuous with other rooted-units, while associated root tissue (all part of the same genet) is laterally continuous - or vice versa (Figure 1b). A RU thus represents a laterally discrete and essential 'life-line' to the substrate for transport of water and mineral nutrients to photosynthesizing tissues (Aarssen 2008). Importantly, for aclonal species, the genet product of a zygote has only one RU to lose, and if lost prior to sex, it fails completely as a vehicle for gene survival/transmission.

The sizes of RUs within a species typically display an enormous range of phenotypic plasticity (for a given age and genotype), and all RUs eventually die (commonly at various sizes). Consequently, the survival of resident genes/alleles (through their copying and transmission success) into the distant future need not be reliably predicted by (and may even be uncorrelated with) the age or size that a particular RU attains. More directly, gene/allele copies are transmitted in perpetuity only because parental RUs (and only entire RUs, not their dependent subunits or modules) make offspring RUs that can in turn make 'grand-offspring' RUs, and so on. The RU thus represents the principal functional vehicle for gene/allele survival (through copying and transmission), and hence the minimal unit for objectively defining - and for enumeration ofindividual sporophyte offspring. RUs normally also make gametophyte 'offspring' derived from spores, but the latter (as well as the sporophyte zygotes and embryos resulting from syngamy within gametophytes) are entirely dependent on provisioning from parental sporophyte RU tissue. In other words, sex (meiosis and syngamy) in seed plants requires that male and female gametophytes receive supporting resources from a sporophyte RU—not vice-versa.

In aclonal species, a parental RU can produce offspring RUs only through production of zygotes/ seeds. In clonal species however, 'daughter' RUs can also be produced through adventitious root or shoot development (Figure 1b), where they are sometimes referred to as 'ramets' (Harper 1977). Ramet (clonal) $\mathrm{RU}$ offspring therefore, may or may not (because of fragmentation) remain interconnected (Figure 1b). The ramet thus extends the somatic longevity of the genet, but in terms of fitness, the ramet RU-like the seedling RU - adds to the copying and transmission success of genes/alleles residing in the parental RU. The collection of grand-offspring RUs for a parental RU may thus be of both types, with some of each grand-offspring RU type (zygotic and clonal) produced by zygotic RU offspring, and some of each type also produced by clonal RU offspring (Figure 2).

These two types of RU offspring and grand-offspring may (or may not) differ in several respects:

Genotypic variability. Outcrossed sexual (seed) RU offspring are of course genotypically variable, whereas clonal (vegetative) RU offspring (like seed offspring from apomixis or from inbred sexual lines) are normally genotypically identical-but not always, owing to somatic mutation (Gill et al. 1995, Fagerstrom et al. 1998, Reusch and Bostrom 2011, Gross et al. 2012).

Dependence on parental $\boldsymbol{R} U$. Clonal RU offspring are, initially at least, physically connected to neighbouring parental RUs, usually with some degree of physiological integration, at least temporarily (Figure 1b). Resource translocation from the parental RU will of course be essential in providing for the early development and establishment of a new daughter RU (Song et al. 2013). But as the latter develops its own local vascular transition point between root tissue and shoot tissue (Figure 1b), translocation from a neighbouring $\mathrm{RU}$ is likely to contribute progressively less to the total resource acquisition by the new daughter RU. Moreover, even regardless of the duration and extent of physiological integration, such clonal RU offspring can be regarded as simply having extended 'maternal care', analogous to the maternal care received by immature zygotic offspring through maternal resources delivered to the embryo and young seedling from connection/ integration with the female gametophyte or endosperm/perisperm of a seed (e.g. consider the massive endosperm of coconuts). In many cycads, establishing seedlings receive nutritional support for several weeks through connection of cotyledons to the female gametophyte within the seed (Chamberlain 1919, Scagel et al. 1982).

Facilitation through physiological integration. Physiological integration with a neighbouring RU of the same clone (again, regardless of duration) can also be 


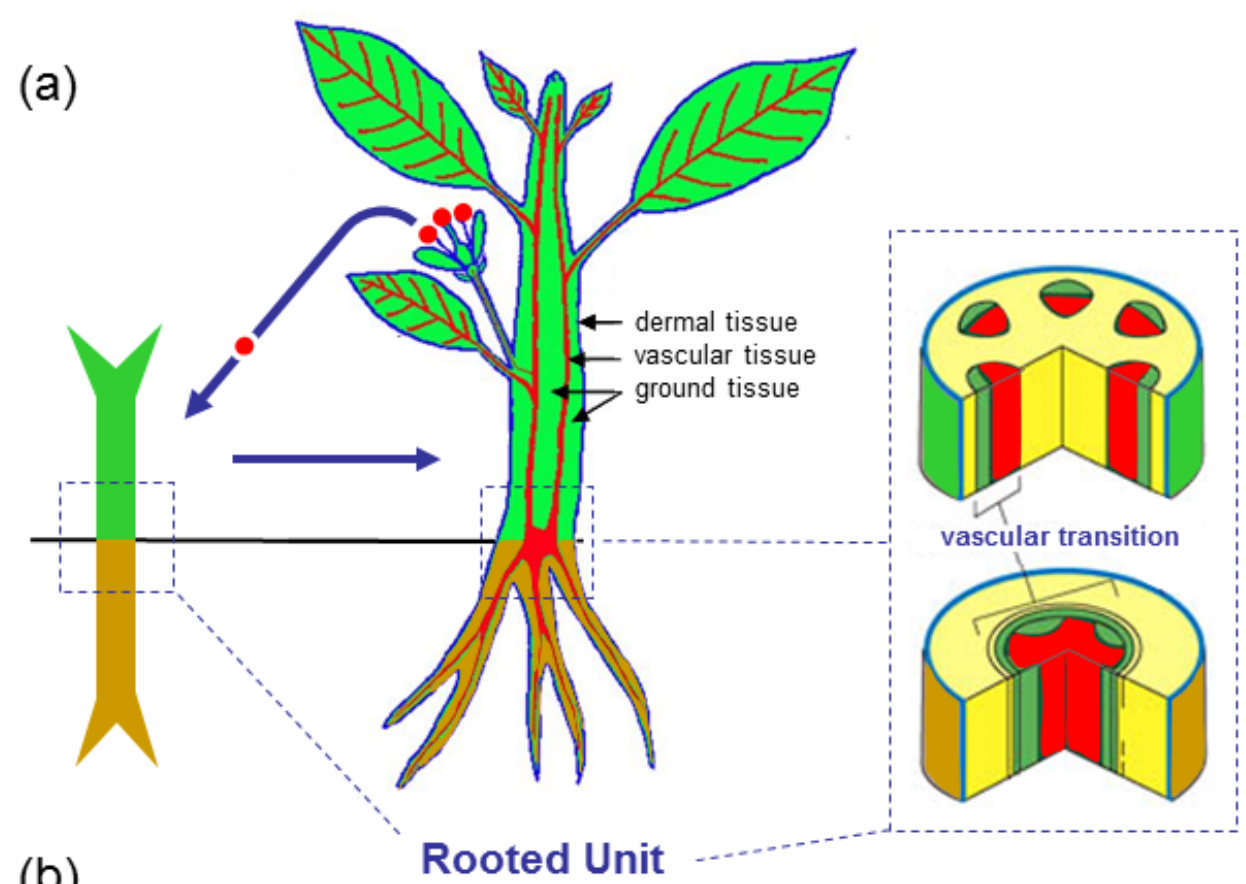

(b)

Rooted Unit

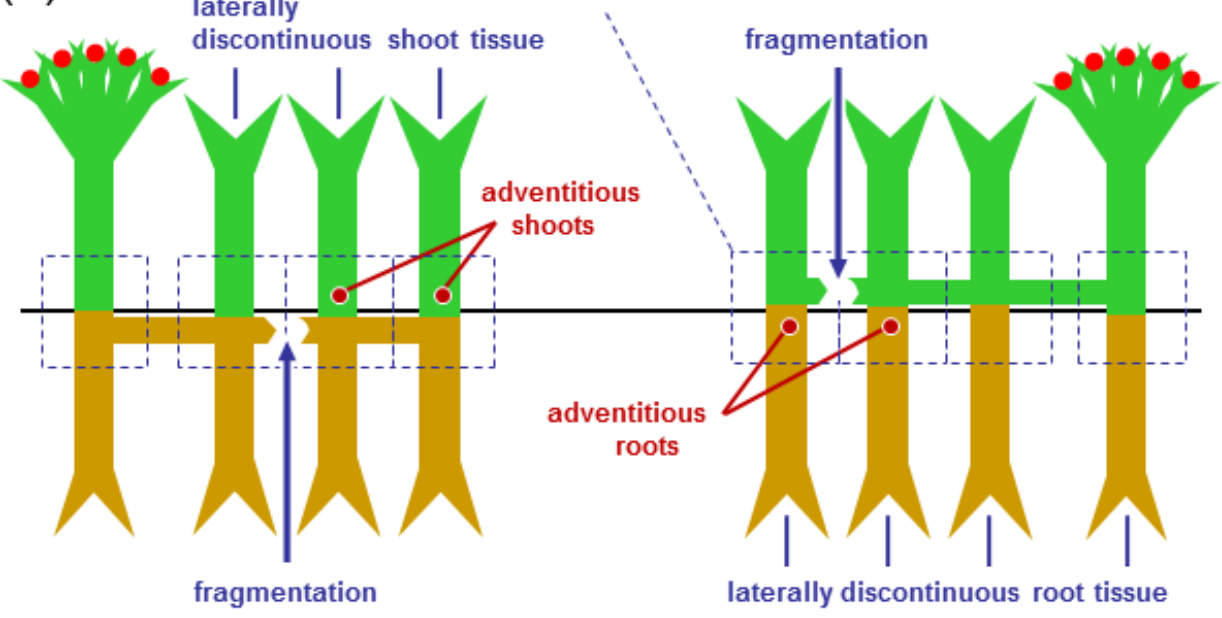

Figure 1. Schematic illustration of a 'rooted-unit' (RU), identified by a distinctly local vascular transition between shoot and root tissue, representing the minimal functional unit for defining an individual sporophyte 'offspring'. In aclonal seed plants (a) a RU can produce offspring RUs only from seeds - i.e. one seed generates exactly (and only) one RU. In clonal plants (b), however, a RU can also produce 'daughter' RUs from adventitious shoot production on lateral roots, or from adventitious root production on lateral shoots. Clonal RU offspring therefore, may or may not (because of fragmentation) remain interconnected. (See text).

regarded as just a particular form of facilitation effect from a neighbour. Aclonal species also commonly benefit from protracted facilitation interactions with neighbouring RUs of both the same and different species (Hunter and Aarssen 1988, Callaway 1995, McIntire and Fajardo 2014), and in some cases this is mediated by physiological integration involving root grafting (Lev-Yadun 2011) or mycorrhizal networks (Selosse et al. 2006), analogous to the physiological integration between clonal RU offspring.

\section{Conspectus}

An individual will, on average, leave no more than one descendant. Because of this, and because of genetic recombination during gamete production, iterated across 


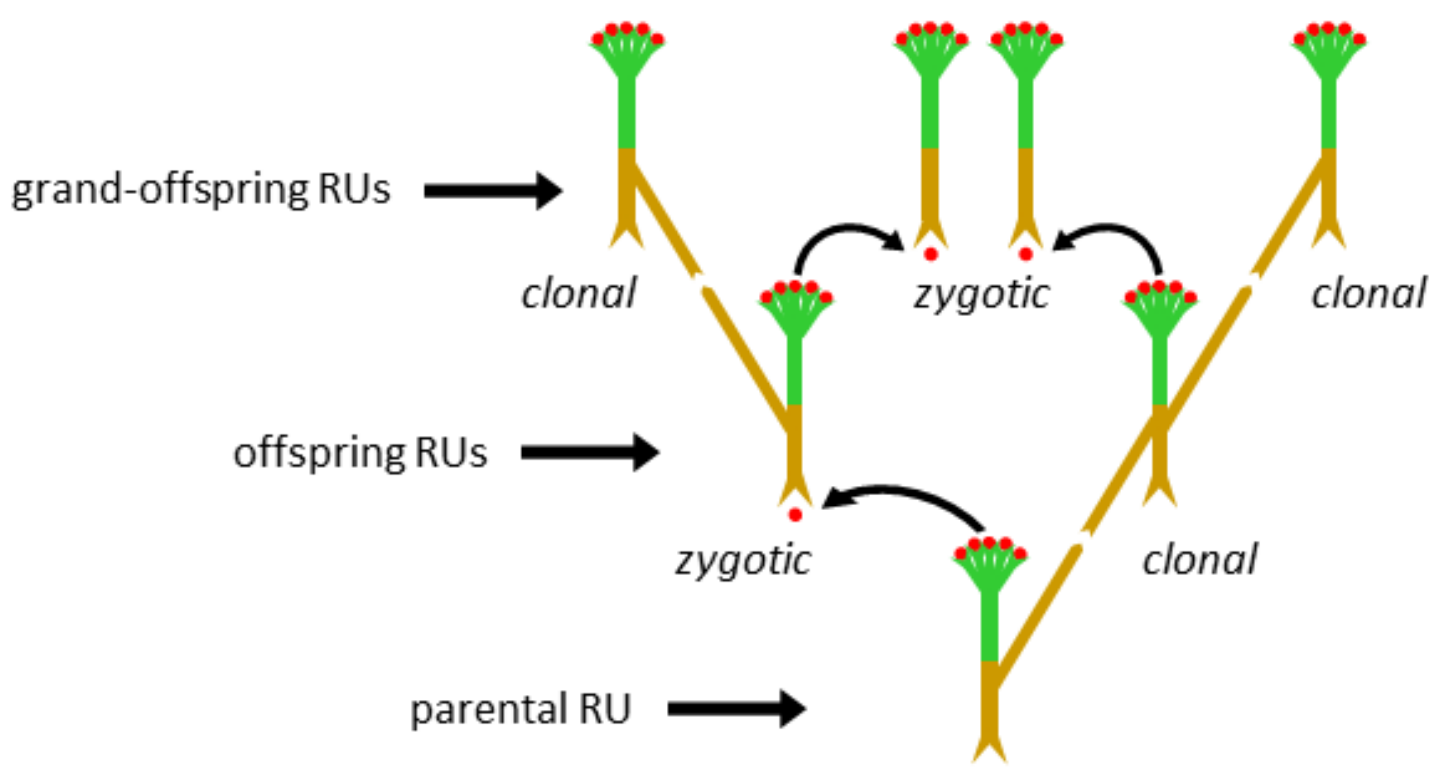

Figure 2. In clonal species, fitness (copying and transmission success of resident genes/alleles) of a parental rootedunit (RU) is estimated by counting both zygotic RU offspring and clonal RU offspring — or more accurately, by counting both zygotic and clonal grand-offspring RUs, where some of each type may have been produced by zygotic RU offspring, and some of each type may also have been produced by clonal RU offspring.

multiple generations, it is highly probable that many (and possible that most or even all) of the living sexual (zygotic) RU descendants of a distant ancestor 'A' will carry not a single gene/allele copy that can be traced back to ' $A$ ' (Dawkins 2004). Accordingly, while selection (differential reproductive success) acts on the phenotypes of RU individuals, it is only their resident genes/alleles (and not these individuals) that realize the effects of selection in terms of fitness, defined by their copying and transmission success across multiple generations (Figure 3). This of course cannot be literally measured, practically - but only estimated, commonly by counting offspring, or descendants only in the short term. In other words, the estimated fitness' of resident genes/alleles for $\mathrm{RU}$ ' $\mathrm{X}$ ' is greater than that for $\mathrm{RU}$ ' $\mathrm{Y}$ ' if the phenotype of ' $\mathrm{X}$ ' (with traits informed by the expression of its resident genes/alleles) evokes greater reproductive success for ' $\mathrm{X}$ ' (i.e. favoured by selection). And importantly, for clonal species, the latter is measured by more than just counting sexual offspring/ descendants (Pedersen and Tuomi 1995, Wikberg 1995).

Both sex (the zygote-zygote cycle) as well as clonality (the meristem-meristem cycle) (Fagerstrom 1990), therefore, can propel gene/allele copies into the future, and thus effect their fitnesses. But critically, in each case, completion of the cycle (within each iteration/generation) requires passing through a rooted-unit stage. And at least one rooted-unit must survive long enough - retaining a functional vascular transition 'lifeline' (Figure 1) - to generate at least one offspring RU through allocating meristem differentiation to sex (meiosis and syngamy), to apomixis, or to clonality. Clonality is likely to be particularly important when the sizes of RUs are severely suppressed (e.g. because of neighbourhood crowding) below their minimum threshold size for successful zygote production through sexual outcrossing (Aarssen 2008).

Accordingly, the rooted-unit is the minimal operational/circumscribable - and hence easily countablevehicle in seed plants that can carry gene/allele copies into the future. And a consensus, I suggest, is possible in recognizing that copying and transmission success (fitness) of resident genes/alleles for a RU is meaningfully estimated by enumeration of offspring RUs derived either zygotically or adventitiously/clonally. Separate counts for each, (where possible) will of course inform regarding important details/predictions for population/genetic structure/dynamics - and for some research objectives, counts of only one or the other may be adequate. In particular, clonal offspring will normally carry more parental gene/allele copies than zygotic offspring. Zygotic offspring, on the other hand, will (if sexually outcrossing) normally carry parental gene/ allele copies that are 'packaged' together with a greater variety of other gene/allele copies transmitted from 


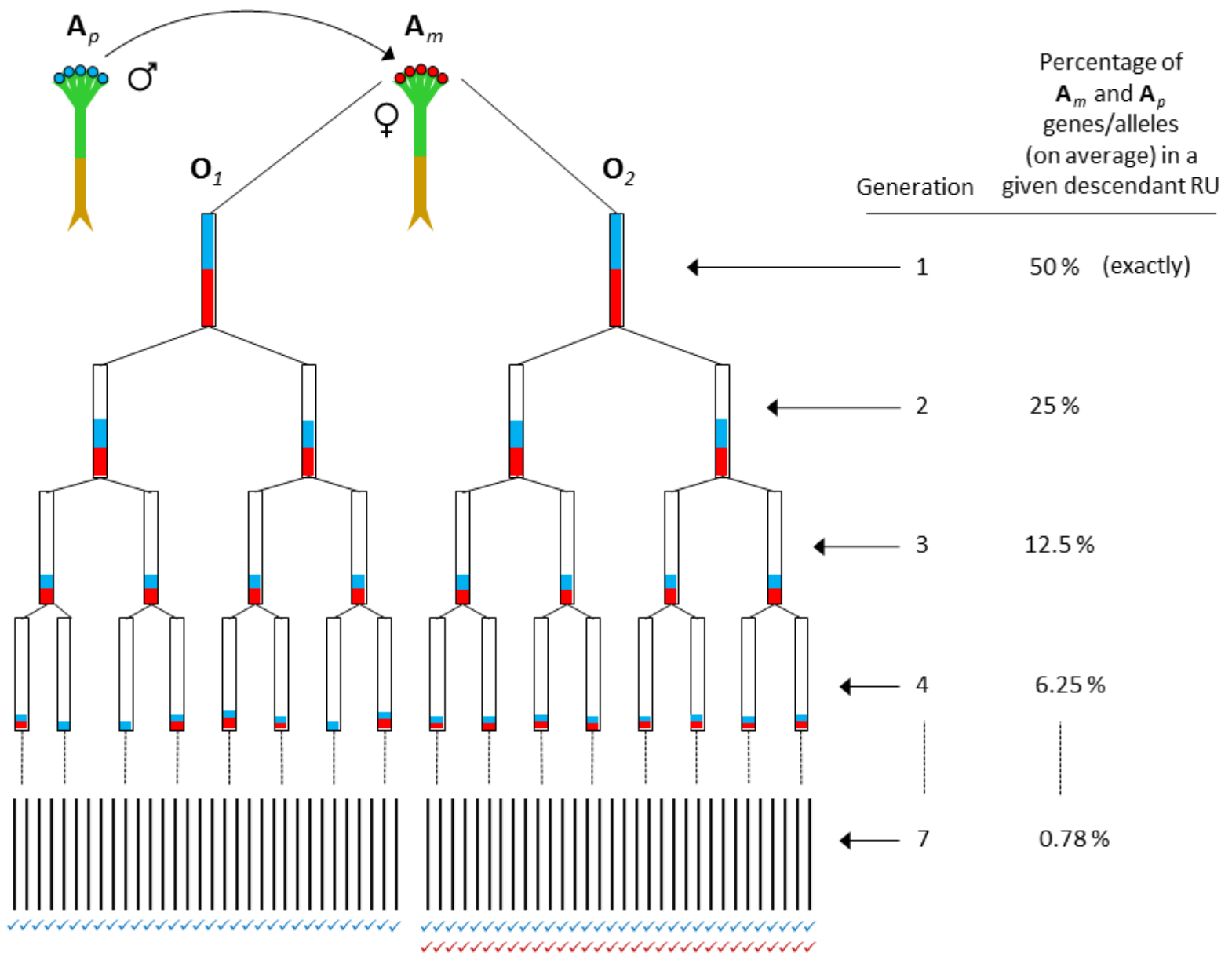

Figure 3. Illustration of why only genes, not individuals, have evolutionary fitness. Counts of descendent RUs for an aclonal obligate outcrossing species, although correlated with gene/allele transmission success in the short term, need not (and may commonly not) be correlated in the long term. In this hypothetical example lineage, ancestral maternal and paternal RUs, $\mathrm{A}_{m}$ and $\mathrm{A}_{p}$, produce two sexual (zygotic) RU offspring, $\mathrm{O}_{l}$ and $\mathrm{O}_{2}$, (represented by vertical rectangles) each of which in turn produce two grand-offspring RUs (generation 2), and so on. Each descendent individual in each generation thus produces two successful reproductive descendants for the next generation (plus additional descendants, not shown because - disfavoured by selection - they fail to reproduce). $\mathrm{O}_{1}$ and $\mathrm{O}_{2}$ each carry copies of exactly $50 \%$ of the resident genes/alleles of both $\mathrm{A}_{m}$ and $\mathrm{A}_{p}$ (indicated by red and blue shading respectively). By generation 7 , the 64 descendants will, on average, carry copies of only $0.78 \%$ of the resident genes/alleles of both $\mathrm{A}_{\mathrm{m}}$ and $\mathrm{A}_{p}$, but some-because of chance during genetic recombination, combined with selection within earlier generations - may carry more or less than this. Importantly, the probability increases with each successive generation that some descendants will carry no gene copies at all, and this is illustrated in the present example for ancestor $\mathrm{A}_{m}$. Thus, $\mathrm{A}_{p}$ and $\mathrm{A}_{m}$ both have the same number of descendants in generation 7—and in fact the same actual descendants in this particular lineage (64 as shown) - and they all carry at least some gene/allele copies inherited from $A_{p}$ (indicated by blue $(\checkmark)$ checks). Yet it is possible, by generation 7 (or perhaps 10 or 20 , but eventually), that only a fraction of the descendants (half shown in this hypothetical example, all descending from $\mathrm{O}_{2}$ ) may carry any gene/allele copies inherited from $\mathrm{A}_{m}$ (indicated by red $(\checkmark)$ checks). Hence, despite $\mathrm{A}_{m}$ having the same descendent RU individuals as $\mathrm{A}_{p}$, resident genes/alleles of $\mathrm{A}_{p}$ have here as much as twofold greater transmission success - presumably, at least partially, because their expression (in combination with other resident genes/alleles within each generation originating from other ancestral RU's, not shown) has informed traits that were more strongly favoured by selection. 
other ancestral RUs, and thus with capacity to display a greater variety of potential phenotypes. Importantly, however, some (perhaps much) of this phenotypic variety will inevitably be disfavoured by selection. When produced together, clonal and zygotic RU offspring thus constitute a bet-hedging strategy, and they do not differ therefore in ways that make one type more legitimate than the other as a counting unit for estimating the fitness of resident genes/alleles. For some species, in fact-where sexual reproduction is virtually dysfunctional or apparently lost within some lineages/ populations (or entirely), e.g. in the Lemnaceae (Vasseur et al. 1992, Eckert 2002) - the only offspring/individuals available to count are clonal. Discounting the latter, when produced (regardless of the level of concurrent sexual prolificacy), will-for many research objectives-underestimate population size, reproductive output/recruitment success within plant populations, and evolutionary fitness of resident genes/alleles.

\section{Acknowledgments}

Thanks to Advisory Editor Carlos Herrera for serving as the handling and decision editor for the manuscript. Helpful comments on the manuscript were provided by Rob Brooker, Chris Eckert, Root Gorelick, Tomas Herben, and Bård Pedersen. Financial support was provided by a Discovery grant from the Natural Sciences and Engineering Research Council of Canada.

\section{Referees}

Bård Pedersen - Bard.Pedersen@ nina.no

Norwegian Institute for Nature Research

Root Gorelick - Root.Gorelick@ carleton.ca Carleton University

\section{References}

Aarssen, L.W. 2008. Death without sex-the problem of the small and selection for reproductive economy in flowering plants. Evolutionary Ecology 22: 279-298. CrossRef

Callaway, R. 1995. Positive interactions among plants. The Botanical Review 61: 306-349. CrossRef

Chamberlain, C.J. 1919. The Living Cycads. University of Chicago Press, Chicago.

Clarke, E. 2012. Plant individuality: a solution to the demographer's dilemma. Biology and Philosophy 27:321-361. CrossRef

Dawkins, R. 1989. The Selfish Gene, rev. ed. Oxford: Oxford University Press.

Dawkins, R. 2004. The ancestor's tale. New York: Houghton Mifflin.
Eckert, C.G. 2002. The loss of sex in clonal plants. Evolutionary Ecology 15:501-520. CrossRef

Fagerstrom, T. 1992. The meristem-meristem cycle as a basis for defining fitness in clonal plants. Oikos 63: 449-453. CrossRef

Fagerstrom, T., Briscoe, D.A., and P. Sunnucks. 1998. Evolution of mitotic cell-lineages in multicellular organisms. Trends in Ecology and Evolution 13: 117-120. CrossRef

Gill, D.E., Perkins, S.L., and J.B. Wolf. 1995. Genetic mosaicism in plants and clonal animals. Annual Review of Ecology and Systematics 26: 423-444. CrossRef

Gorelick, R. 2012. Mitosis circumscribes individuals; sex creates new individuals. Biology and Philosophy 27:871-890. CrossRef

Gross, C.L., Nelson, P.A., Haddadchi, A., and M. Fatemi. 2012. Somatic mutations contribute to genotypic diversity in sterile and fertile populations of the threatened shrub, Grevillea rhizomatosa (Proteaceae). Annals of Botany 109: 331-342. CrossRef

Harper, J.L. 1977. Population Biology of Plants. London: Academic Press.

Hunter, A., and L.W. Aarssen. 1988. Plants helping plants. Bioscience 38: 34-40. CrossRef

Lev-Yadun, S. 2011. Why should trees have natural root grafts? Tree Physiology 31: 575-578. CrossRef

McIntire, E.J.B., and A. Fajardo. 2014. Facilitation as a ubiquitous driver of biodiversity New Phytologist 201: 403-416. CrossRef

Mogie, M. 1992. The Evolution of Asexual Reproduction in Plants. London: Chapman \& Hall, 276 pp.

Pan, J.P., and J.W. Price. 2002. Fitness and evolution in clonal plants: the impact of clonal growth. Evolutionary Ecology 15: 583-600. CrossRef

Pedersen, B., and J. Tuomi. 1995. Hierarchical selection and fitness in modular and clonal organisms. Oikos 73: 167-180. CrossRef

Reusch, T.B.H., and C. Bostrom. 2011. Widespread genetic mosaicism in the marine angiosperm Zostera marina is correlated with clonal reproduction. Evolutionary Ecology 25: 899-913. CrossRef

Scagel, R.F., Bandoni, R.J., Maze, J.R., Rouse, G.E., Schofield, W.B., and J.R. Stein. 1982. Plants: an evolutionary survey. Wadsworth, Belmont CA.

Selosse M-A., Richard, F., He, X., and W. Simard. 2006. Mycorrhizal networks: des liaisons dangereuses? Trends in Ecology and Evolution 21: 621-628. CrossRef

Song Y-B., Yu, F-H., Keser, L.H., Dawson, W., Fischer, M., Dong, M., and M. van Kleunen. 2013. United we stand, divided we fall: a meta-analysis of experiments on clonal integration and its relationship to invasiveness. Oecologia 171: 317-327. CrossRef 
Vasseur, L., Aarssen, L.W., and T. Bennett. 1993. Allozymic variation in local apomictic populations of Lemna minor (Lemnaceae). American Journal of Botany 80: 974-979. CrossRef

Wikberg, S. 1995. Fitness in clonal plants. Oikos 72: 293-397. CrossRef

\section{Response to referee}

The aim of my commentary was to start a conversation that might help enlighten the unresolved debate regarding the identification of legitimate 'counting units' for estimating evolutionary fitness in seed plants. With Gorelick's (2014) insightful response paper, we have a conversation, but it is clear that a consensus is not imminent, and perhaps not even reasonable to expect (especially if there is a lot of drifting away on 'alcoholic wings', which sounds good to me!). Gorelick (2014) offers interesting perspectives on a number of topics, including some that go beyond the scope of my commentary, echoing his earlier published contributions-i.e. concerning the identification of different sources (and their relative amounts) of genetic/genomic variation, and considerations of the relative importance of mutation rate, drift and selection in the evolution of clonal plants. Gorelick also raises several points that give opportunity for me to provide (below) what should be helpful clarification on some important issues.

Gorelick writes that my "...definition of clonality and individuals is dependent on identifying rooted-units that are characterized by root-to-shoot transitions in the stele"; and that because of this, "Monocots may be problematic for Aarssen's proposed rooted-unit circumscription of individuals". Actually, I said that rooted-units are "normally" (not universally) characterized as such. And more importantly (to reiterate my definition in the original text), the essential distinction of clonal rooted-units obtains when the somatic product of a zygote involves root tissue or shoot tissue (or both) that is laterally discontinuous. Each root-shoot transition for such a plant thus defines a 'rooted-unit' and this may or may not (but commonly does) involve distinctly different above- versus below-ground stele anatomy/ architecturre. The monocots as a group may indeed have uniform stele configurations above- and below-ground (and I was not aware of this possibility), but this does not confound the definition of individuals/offspring as rooted-units, nor is it problematic for counting clonal rooted-units in estimations of fitness.

Gorelick correctly points out that, in some species, leaves or leaf cuttings (not just stems or roots) may form adventitious roots and shoots and thus become separate ramets. But he claims (incorrectly) that this presents "... a problem with Aarssen's circumscription of clones and individuals as rooted-units." I had overlooked this specialized mechanism for generating clonal rootedunits from above-ground somatic tissue. However, these 'clonal leaves' - in addition to detachable axillary and inflorescence bulbils, as well as detachable stems (e.g. in Opuntia spp) (all serving as dispersible clonal propagules)_nevertheless develop into rooted-units. And importantly here, if they fail to develop into rooted units (and many/most will), they fail as carriers of gene copies into the future - which necessarily means that the rooted unit (and not these detachable modular precursors) is indeed the minimal operational and circumscribable 'vehicle.' It also remains true that in order to produce these dispersible vegetative subunits capable of becoming daughter RUs, the entire (fully functional) 'parental' RU must survive long enough to do so. Accordingly, the central argument - that a functional and practical circumscription for enumerating individual 'offspring' applies equally to rooted-units derived from zygotes, or from clonality-is not compromised.

Finally, Gorelick (as in earlier literature) takes issue with naming apomictic seeds as individual offspring. In response, I can only reiterate again my central argument-that all seeds, whether amphimictic or apomictic, have potential to develop into separate circumscribable rooted-units. By virtue of this then, they represent (along with vegetative/clonal RUs) legitimately countable individual offspring in the estimation of fitness (transmission success) for the resident genes/alleles within parental RUs. And this conclusion arrives because only individual genes/alleles (and not 'individuals' of any other variety), have evolutionary fitness (Figure 3).

Gorelick, R. 2014. Defining clonality and individuals in plant evolution. Ideas in Ecology and Evolution 7: 8488. CrossRef 